\title{
Cancer therapy and cardiovascular risk: focus on bevacizumab
}

\author{
This article was published in the following Dove Press journal: \\ Cancer Management and Research \\ 3 June 2015 \\ Number of times this article has been viewed
}

\section{Panagiota Economopoulou' \\ Athanasios Kotsakis ${ }^{2}$ \\ loannis Kapiris ${ }^{3}$ \\ Nikolaos Kentepozidis ${ }^{3}$}

'Medical Oncology Unit, 2nd Department of Internal Medicine, Attikon University Hospital, Haidari, Athens, ${ }^{2}$ Department of Medical Oncology, University Hospital of Heraklion, Crete, ${ }^{3} 25$ I Airforce General Hospital, Department of Medical Oncology, Athens, Greece

Correspondence: Panagiota Economopoulou

Medical Oncology Unit, 2nd Department of Internal Medicine, Attikon University Hospital, National Kapodistrian University of Athens, Ist Rimini St, 12462, Haidari, Athens, Greece Tel +302105831655

Fax +302105831690

Email panagiota_oiko@hotmail.com

\begin{abstract}
Recognition and management of treatment-related cardiovascular toxicity, defined as either an acute cardiac event or a chronic condition, has been tightly integrated into routine cancer care and has become an important component in treatment selection. Several chemotherapeutic agents, such as anthracyclines, are traditionally characterized as cardiotoxic, but cardiovascular adverse events are also associated with commonly used molecular targeted therapies. In the past decade, bevacizumab, a monoclonal humanized antibody against vascular endothelial growth factor, has been introduced in the treatment of a variety of metastatic malignancies. Despite its efficacy, bevacizumab has been associated with significant risk of cardiovascular complications, such as hypertension, cardiac ischemia, and congestive heart failure. This review will focus on the cardiovascular toxicity of bevacizumab, providing the latest evidence on the incidence, clinical spectrum, risk factors, and responsible mechanisms.
\end{abstract}

Keywords: bevacizumab, cardiovascular toxicity, hypertension

\section{Introduction}

Systemic anticancer treatments can have detrimental effects on the cardiovascular system, either by exerting their own toxic effects or by augmenting side effects of other drugs. ${ }^{1}$ As the development of novel drugs evolves, cancer survival has improved and cardiac toxicity caused by various anticancer agents has greater potential impact on long-term outcomes. The emerging field of cardio-oncology has developed strategies to minimize cardiovascular toxicity and prevent long-term effects.

Cardiotoxicity includes acute events, such as arrhythmias, myocardial ischemia, vasospastic and thromboembolic ischemia, pericarditis and/or myocarditis-like syndromes, and chronic conditions, such as left ventricular (LV) dysfunction (LVD) with or without overt congestive heart failure (CHF), arterial hypertension (HTN), and QTc prolongation. ${ }^{2}$ More specifically, according to the Cardiac Review and Evaluation Committee, LVD is characterized by the following: 1) a decrease in cardiac LV ejection fraction (LVEF) that is either global or more severe in the septum; 2) symptoms of $\mathrm{CHF}$; 3 ) signs of $\mathrm{CHF}$, including but not limited to S3 gallop, tachycardia, or both; and 4) decreases in LVEF from baseline of at least 5\% to below 55\% with associated signs or symptoms of CHF, or at least $10 \%$ to below $55 \%$ without associated signs or symptoms. ${ }^{3}$

Anticancer drugs that induce cardiotoxicity have been divided into two categories depending on the reversibility of myocardial damage. ${ }^{4}$ Type I agents directly cause cell death leading to irreversible myocyte destruction and clinical CHF. These include traditional anticancer therapies, such as anthracyclines, alkylating agents, and 
antimicrotubule agents. On the other hand, type II agents alter normal cellular function by affecting the mitochondrial system and reducing protein synthesis, which is reversible once the drug is discontinued. Type II cardiotoxicity was first described with trastuzumab, although more recently, it has been associated with newer targeted therapies, including vascular endothelial growth factor (VEGF) inhibitors and tyrosine kinase inhibitors. ${ }^{5}$

Bevacizumab is a humanized monoclonal antibody against the VEGF-A ligand that binds to its circulating target, altering the kinetics of ligand binding to endothelial cells and downregulating angiogenesis. ${ }^{6}$ It has been approved by the European Medicines Agency and/or by the United States Food and Drug Administration, and it is the first- or secondline chemotherapy for the treatment of many advanced solid tumors, including colorectal cancer (CRC), non-small-cell lung cancer (NSCLC), breast cancer, glioblastoma, renal cell cancer (RCC), ovarian cancer, and cervical cancer. ${ }^{7-14}$ Although the efficacy of bevacizumab has been demonstrated in many clinical trials, its use has been associated with many cardiovascular events, such as high-grade HTN and thromboembolism. ${ }^{15}$

The aim of this review is to summarize and discuss the available evidence on the cardiovascular toxicity of anticancer systemic therapies, with special attention paid to the recently recognized adverse effects of bevacizumab. In the era of personalized medicine, knowing the potential cardiovascular risks of anticancer agents might influence the optimal choice of treatment and allow for the establishment of prevention strategies.

\section{Cardiovascular toxicity of bevacizumab}

In several trials evaluating the efficacy and toxicity of bevacizumab, its use has been predominantly complicated with HTN, CHF, and thromboembolic events. The incidence of the cardiovascular toxicity of bevacizumab in important clinical trials is summarized in Table 1.

\section{HTN}

HTN is a common adverse event occurring in patients treated with bevacizumab, with an overall incidence of 4\%-35\% reported in clinical trials. ${ }^{9,10,16,17}$ This variability might be attributed to the different selection criteria used in clinical trials (eg, the age of the patients included), as well as to differences in the definition of HTN. The mechanism of bevacizumab therapy-related HTN is not fully understood. It has been proposed that VEGF inhibition decreases nitric oxide production in the endothelium, leading to vasoconstriction and, therefore, to increased peripheral vascular resistance and blood pressure (BP). ${ }^{18}$ Reduced levels of nitric oxide promotes the expression of plasminogen-activator inhibitor 1 (PAI-1), resulting in exacerbation of HTN. Furthermore, VEGF inhibition has been associated with cholesterol embolization syndrome, which refers to embolization of the contents of an atherosclerotic plaque from a proximal largecaliber artery to distal small arteries. This leads to a multitude of small emboli occurring over time, causing mechanical plugging of the arteries and HTN. ${ }^{19}$

In Phase I trials, bevacizumab was safely administered at a dose up to $10 \mathrm{mg} / \mathrm{kg}$ without dose-limiting toxicities, but mild increases in BP were observed at higher dose levels tested. ${ }^{20}$ In Phase II trials assessing the efficacy and toxicity of the drug, severe HTN (grade 3 and 4) was reported in $9 \%-15 \%$ of patients. $^{21-23}$ On the other hand, in Phase II trials that evaluated bevacizumab in combination with other chemotherapy agents or targeted therapies, grade 3 or 4 HTN ranged from $0 \%-19 \% .{ }^{24-35}$ As expected, patients who crossed over from the control arm to the bevacizumab arm also displayed HTN. The median interval from initiation of bevacizumab to the development of HTN is approximately 4.6-6 months. In the majority of Phase III trials that led to the approval of bevacizumab, HTN was statistically significant more frequently in the bevacizumab-treated arm. ${ }^{8-11,13,14}$ In a landmark Phase III trial that assessed the efficacy of bevacizumab alone or in combination with irinotecan in recurrent glioblastoma, HTN was more frequent in the bevacizumab alone arm. ${ }^{12}$ In a recent meta-analysis of randomized controlled trials, the addition of bevacizumab to chemotherapy was associated with a statistically significant increase in high BP. Interestingly, patients with RCC and breast cancer who received the drug at a dose of $5 \mathrm{mg} / \mathrm{kg}$ weekly had a higher risk of developing HTN. ${ }^{36}$

Bevacizumab-related HTN can develop at any time during treatment, and the data suggest that there is a dose relationship. ${ }^{37}$ More specifically, the risk of HTN is increased by three times with low doses and 7.5 times with high doses of bevacizumab. ${ }^{38}$ Most patients who developed HTN in clinical trials were treated with antihypertensive medication and continued bevacizumab. This is particularly important, since there is a clear association between the efficacy of and duration of exposure to bevacizumab. ${ }^{39}$ However, HTN resistant to medication might lead to discontinuation of bevacizumab in $1.7 \%$ of patients. ${ }^{1}$ Single cases of hypertensive crisis with encephalopathy and subarachnoid hemorrhage have also been reported. ${ }^{1}$ 
Interestingly, several clinical trials have shown that the development of bevacizumab-related HTN is a predictive marker for clinical outcomes. Reported data from various malignancies, such as metastatic breast, metastatic colorectal, non-small-cell, and ovarian cancer, as well as malignant glioblastoma, clearly indicate an improved progression-free survival and/or overall survival in patients who develop HTN as a drug side effect. ${ }^{40-45}$ The responsible underlying mechanism has not yet been clarified.

The exact factors that predispose an individual to bevacizumab-induced HTN have not been established. However, several risk factors associated with VEGF inhibitor (VEGFI)-related HTN have been identified, including a previous history of HTN, age $>65$ years, smoking, and possibly hypercholesterolemia. ${ }^{46}$

For the treatment of bevacizumab-related HTN, standard hypertensive medications are used, according to the European Society of Cardiology (ESC) and European Society for Medical Oncology (ESMO) guidelines. ${ }^{47-50}$ It is important not to withdraw treatment early, but rather to implement active antihypertensive medication with the objective of obtaining a $\mathrm{BP}<140 / 90 \mathrm{mmHg}$. A combination of antihypertensive drugs might be required, and close monitoring of $\mathrm{BP}$ is mandatory. Angiotensin-converting enzyme (ACE) inhibitors are suggested as the first-line treatment, since they have been shown to prevent proteinuria (also induced by bevacizumab) and PAI-1 expression. ${ }^{51}$ Furthermore, in vivo studies have demonstrated that ACE inhibitors increase the release of the natural vasodilator nitric oxide, overcoming the proposed mechanism of bevacizumab-associated HTN. ${ }^{52}$ However, they seem to have suboptimal BP-lowering effects when HTN is severe. In that case, calcium channel blockers are particularly effective, possibly because they reduce vascular smooth muscle cell contraction in vessels that are hypercontractile due to VEGFI-induced impairment of nitric oxide signaling. Only dihydropyridine calcium channel blockers, such as amlodipine or nifedipine, should be used, because nondihydropyridine calcium channel blockers, such as diltiazem or verapamil, inhibit cytochrome P450 3A4, which metabolizes VEGFIs, thus leading to potentially high levels of plasma bevacizumab..$^{53}$ Discontinuation of bevacizumab may be applicable if systolic BP is $>200 \mathrm{mmHg}$ or if diastolic $\mathrm{BP}$ is $>100 \mathrm{mmHg}$, or in cases of hypertensive crisis.

\section{$\mathrm{CHF}$}

Approximately $2 \%-4 \%$ of patients treated with bevacizumab will develop CHF. ${ }^{1}$ Predisposing factors include previous therapy with cardiotoxic chemotherapy drugs, such as anthra- cyclines $^{24}$ and capecitabine, ${ }^{10}$ as well as irradiation to the mediastinum. ${ }^{1}$ The main mechanism responsible for bevacizumab-associated CHF is suggested to be uncontrolled HTN, leading to LV hypertrophy. ${ }^{54}$ On the other hand, animal studies have demonstrated that normal cardiac growth and preserved contractile function are associated with enhanced coronary angiogenesis; thus, disruption of coordinated tissue growth and angiogenesis in the heart, induced by bevacizumab, contributes to progression from adaptive cardiac hypertrophy to heart failure (HF). ${ }^{55}$ Furthermore, angiogenesis plays a key role in the normal adaptive response to pressure overload. A study that has utilized strategies mimicking the mechanism of bevacizumab have shown that pressure overload resulted in a reduction of contractile dysfunction and eventually decompensated HF. ${ }^{54}$

CHF has mainly been reported in clinical trials assessing the efficacy and toxicity of bevacizumab in breast cancer patients. This might be related to the fact that the majority of patients with metastatic breast cancer have been previously treated with cardiotoxic drugs, such as anthracyclines. In a Phase III breast cancer trial, anthracycline treatment preceded all cases of cardiomyopathy and HF (2.6\% of patients). ${ }^{56} \mathrm{In}$ another study, ${ }^{10} \mathrm{LVD}$ was reported in $<1 \%$ of patients. On the contrary, no cases of HF were reported in colorectal or lung cancer trials evaluating bevacizumab. ${ }^{7,9,57}$ In a recent retrospective study that included 6,937 patients aged $\geq 65$ years with CRC, no association between bevacizumab and CHF or cardiac death was observed. ${ }^{58}$ These results suggest that the main predisposing factor for the development of CHF in patients receiving bevacizumab is not advanced age, but rather previous therapy with cardiotoxic drugs.

Cancer patients on bevacizumab therapy who develop HF should be treated according to guidelines proposed by the ESC and ESMO ${ }^{50,59}$ Patients developing asymptomatic LVEF dysfunction of $<50 \%$ during bevacizumab therapy should be referred to a cardiologist and receive ACE inhibitors. Bevacizumab should be stopped until improvement and normalization of LVEF. Patients with more advanced stages of HF should receive a combination of ACE inhibitors and beta blockers, unless contraindicated. As HF worsens, additional medication, such as diuretics, is required. ${ }^{60} \mathrm{All}$ patients with HF should undergo coronary angiography to exclude coronary artery disease.

\section{Arterial thromboembolic events (ATEs)}

Combination treatment with bevacizumab and chemotherapy is associated with an increased risk of arterial thromboembolism (myocardial and cerebrovascular events). 


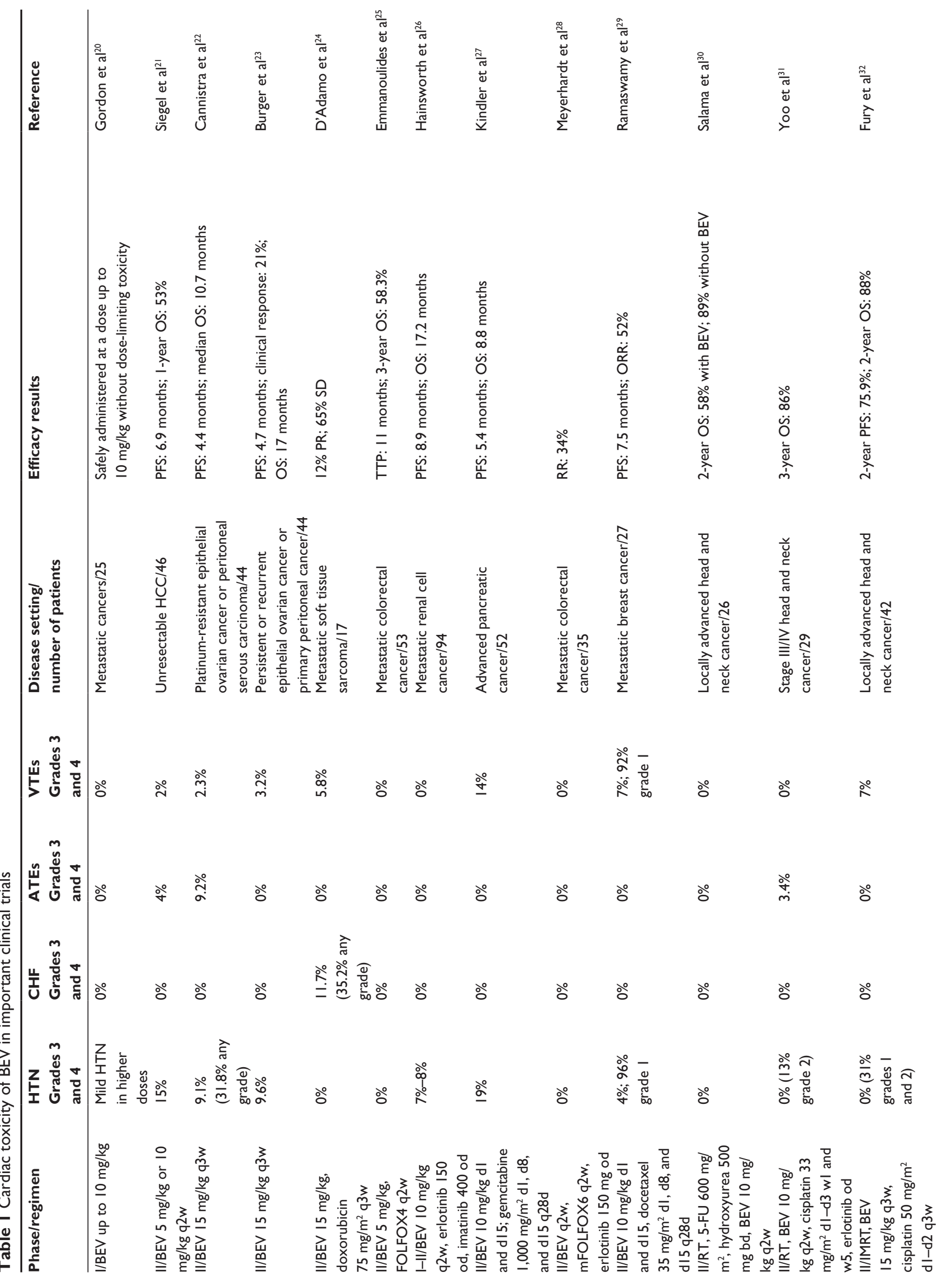




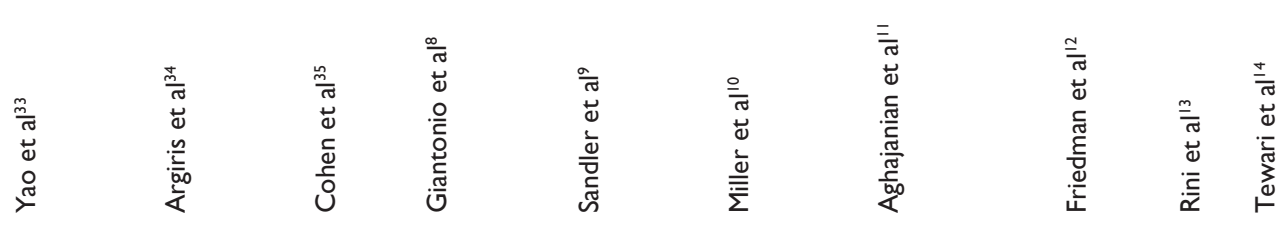

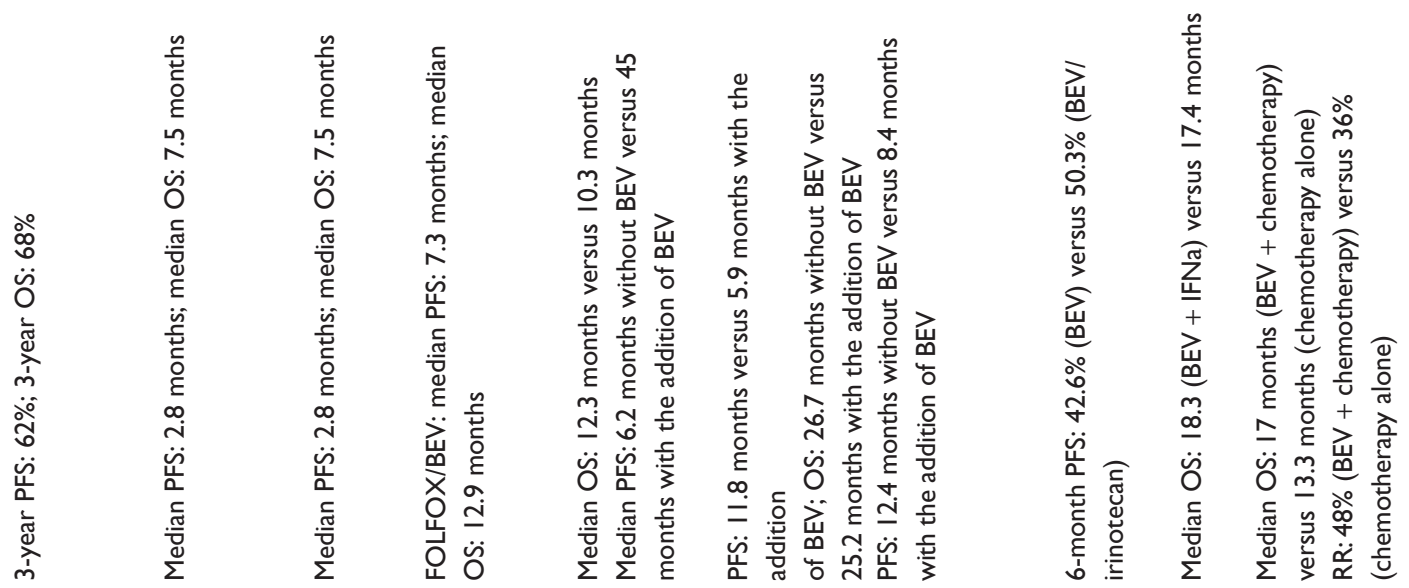

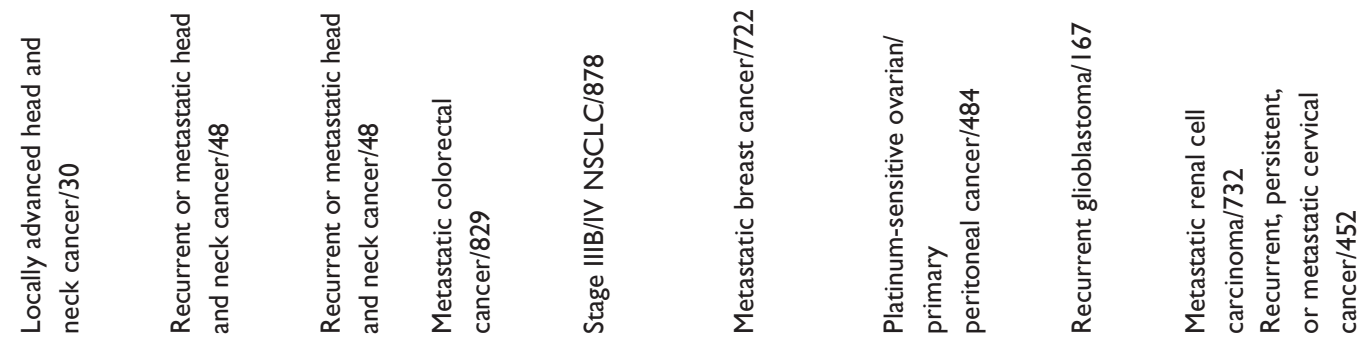

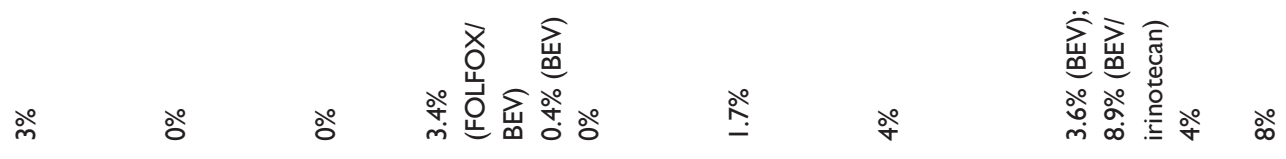

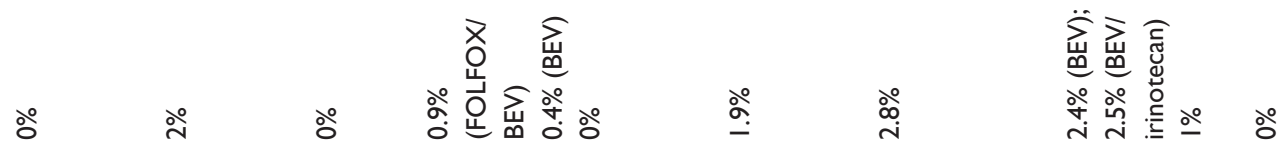

○ ㅇ

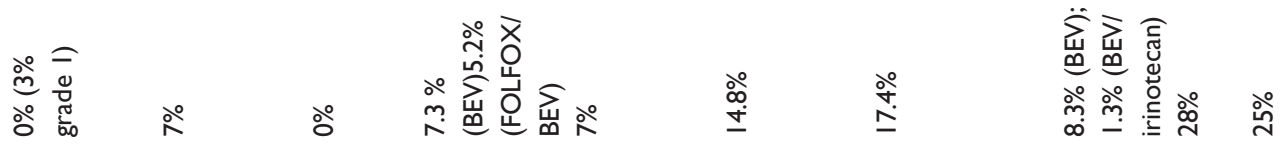

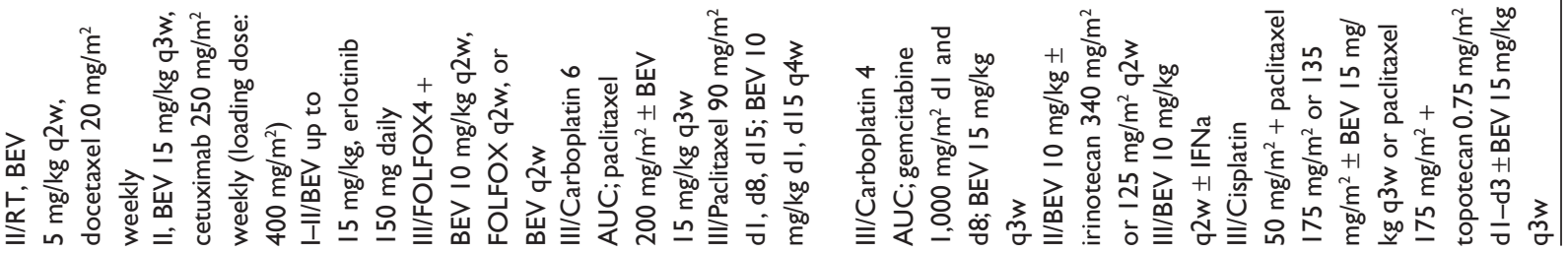




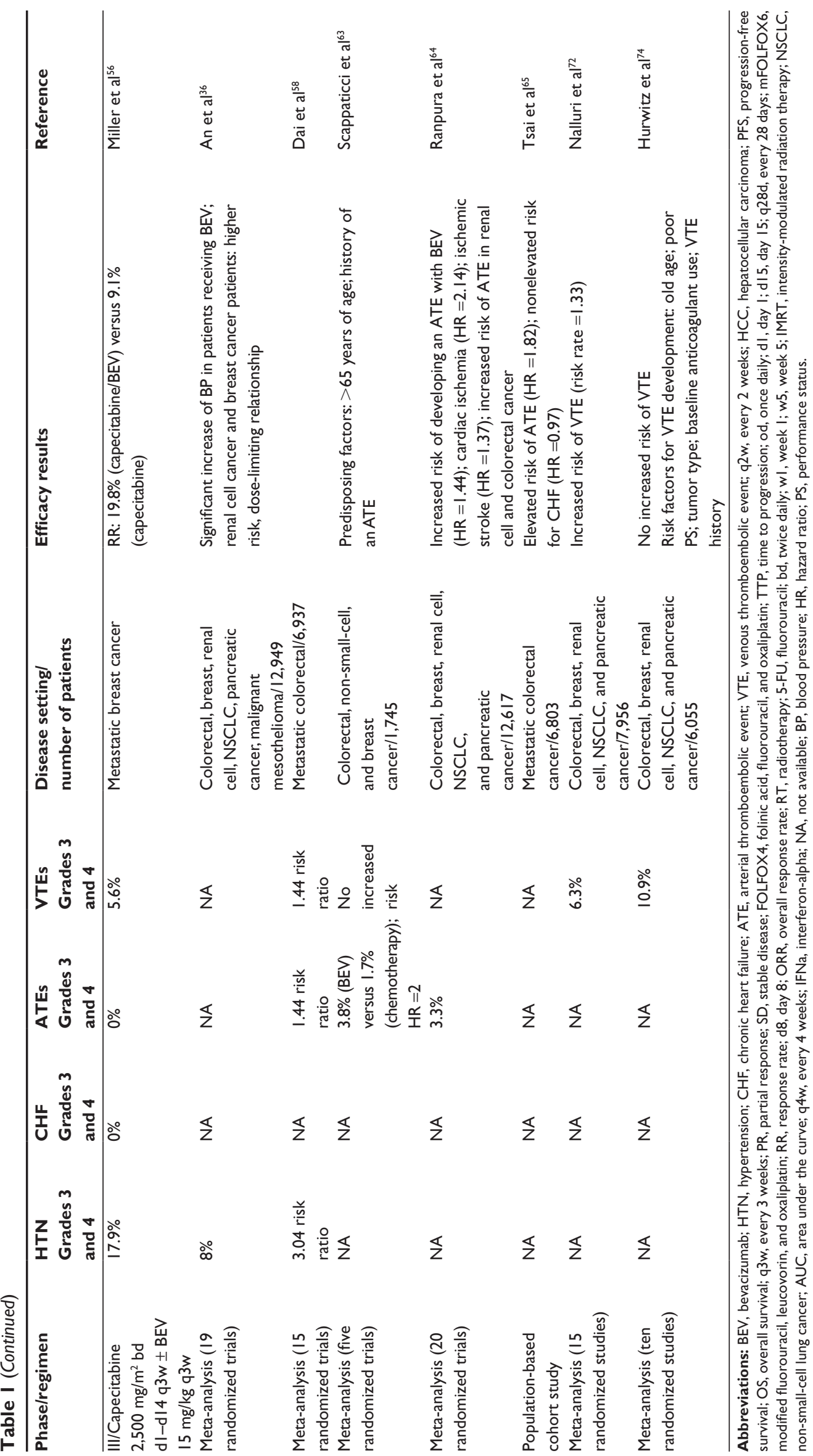


The responsible underlying mechanism remains unclear. It is well known that the characteristic feature of any ATE is the instability of atherosclerotic plaques and the associated activation of platelets. Bevacizumab might reduce antiinflammatory effects of chronic VEGF exposure, leading to increased inflammation and atherosclerotic instability, and to subsequent plaque rupture and thrombus formation. ${ }^{61}$ Additionally, VEGF is important for the proliferation and repair of endothelial cells. ${ }^{18}$ Therefore, anti-VEGF therapy may decrease the regenerative capacity of endothelial cells in response to trauma, leading to endothelial cell dysfunction and exposing subendothelial collagen. As a result of subendothelial collagen exposure, the tissue factor is activated, increasing the risk of thrombosis. ${ }^{18,62}$ Finally, anti-VEGF therapy causes a reduction in nitric oxide and prostacyclin, as well as an increase in blood viscosity via the overproduction of erythropoietin, all of which comprise predisposing factors for increased risk of thromboembolic events. ${ }^{62}$

A pooled analysis of five randomized trials in metastatic CRC, NSCLC, and breast cancer that involved a total of 1,745 patients, demonstrated a higher risk of developing an ATE (such as angina, myocardial or cerebral ischemia/infarct and arterial thrombosis) in patients treated with chemotherapy and bevacizumab, as compared to those treated with chemotherapy alone $(3.8 \%$ vs $1.7 \%$ in the control group; $P<0.05$; hazard ratio $=2) .{ }^{63}$ The absolute rate of developing an ATE was 5.5 events per 100 patient-years for patients receiving chemotherapy plus bevacizumab, as compared to 3.1 events per 100 patient-years for those receiving chemotherapy alone (relative rate $[\mathrm{RR}]=1.8 ; 95 \%$ confidence interval [CI]: 0.94-3.33). When looking at myocardial infarction/ angina specifically, the incidence was $1.5 \%$ versus $1 \%$ in the bevacizumab group as compared with the control group, respectively. Predisposing factors were found to be old age ( $>65$ years) and a history of an ATE. ${ }^{63}$ A more recent metaanalysis, which included 12,617 patients from 20 Phase II and III randomized controlled trials, had similar results, showing a significantly increased risk of ATEs in patients receiving bevacizumab, as compared to controls $(\mathrm{RR}=1.44$; 95\% $\mathrm{CI}$ : 1.08-1.91). ${ }^{64}$ More specifically, bevacizumab was associated with a significantly increased risk of cardiac ischemia ( $\mathrm{RR}=2.14$; 95\% CI: 1.12-4.08), but not stroke (RR =1.37; 95\% CI: 0.67-2.79, $P=0.39)$. Patients receiving bevacizumab had an overall incidence of all-grade ATEs of 3.3\%, whereas the incidence of high-grade events was $2 \%$. As opposed to bevacizumab-related HTN, a dose-effect relationship was not found for the risk of ATEs, which was similar for doses of $2.5 \mathrm{mg} / \mathrm{kg} /$ week and $5 \mathrm{mg} / \mathrm{kg} /$ week. Furthermore, the risk varied with the type of malignancy, with the highest risk of all-grade ATEs demonstrated in patients with CRC (6.1\%; 95\% CI: 4.4-8.5); bevacizumab significantly increased that risk $(\mathrm{RR}=2.79 ; 95 \% \mathrm{CI}: 1.42-5.49) .{ }^{64}$ On the other hand, in a recent observational study that involved 6,803 elderly patients with CRC, patients treated with bevacizumab had a modestly elevated risk of ATEs; however, this did not commonly produce a clinical impact when expressed in absolute terms (four additional ATE cases per 1,000 person-years). ${ }^{65}$ In Phase II trials assessing bevacizumab in combination with other agents in head and neck cancer, cardiac ischemia was reported in one patient in two studies; ${ }^{31,34}$ another study showed a relatively high incidence of syncope $(7 \%){ }^{32}$ In all studies mentioned, bevacizumab-associated ATEs were reported to occur at any time during therapy. ${ }^{63-65}$

Patients with suspected cardiac ischemia should be managed according to the guidelines established by the ESC and ESMO. ${ }^{50,66,67}$ However, one major concern in cancer patients treated with bevacizumab is the use of antiplatelet and anticoagulant therapy, due to increased risk of bleeding. Currently, there are no guidelines or prospective studies that include such patients. However, in the previously mentioned metaanalysis, concomitant use of chemotherapy, bevacizumab, and aspirin did not substantially increase the risk of bleeding compared to the use of aspirin and chemotherapy alone, and aspirin-based prophylaxis for an ATE is recommended for all cancer patients at risk when these is no contraindication. ${ }^{63}$ Furthermore, aspirin use has been shown to improve survival in cancer patients with cardiac ischemia, irrespective of thrombocytopenia. ${ }^{68}$ On the other hand, because studies excluded patients who had any history of stroke or myocardial infarction within 12 months of enrollment, the risks and benefits of bevacizumab treatment among these patients have not been established. Bevacizumab therapy should be discontinued in patients who develop severe ATEs during treatment; the safety of restarting bevacizumab therapy after resolution of an ATE has not yet been studied.

\section{Venous thromboembolic events (VTEs)}

The role of bevacizumab in the development of VTEs is controversial. In initial Phase II and Phase III clinical trials, the reported incidence of VTEs varied from 3\%-19.4\%. ${ }^{12,69,70}$ Several other Phase III randomized trials revealed a higher risk of VTEs associated with bevacizumab, although this was not statistically significant compared to controls. ${ }^{8,10,71}$ In the previously mentioned meta-analysis by Scappaticci et al, ${ }^{63}$ bevacizumab therapy did not alter the risk of a VTE, and based on those results, venous thromboembolism was 
not considered a major adverse event of bevacizumab. However, a meta-analysis conducted in 2008 that included 7,956 patients from 15 randomized trials with a variety of solid tumors (breast, colorectal, non-small-cell lung, renal cell, and other cancers) identified a significantly elevated risk of VTEs associated with bevacizumab therapy $(\mathrm{R} R=1.33$; 95\% CI: $1.13-1.56 ; P<0.001)$; this risk was observed in allgrade and high-grade VTEs. ${ }^{72}$ Similar to ATEs, the incidence of bevacizumab-related VTEs did not have a dose-effect relationship. A higher risk was found in lung cancer. ${ }^{72}$ This meta-analysis received criticism mainly focused on the fact that it was based on summary rates than on individual patient data, and there was no adjustment for differential observation times. ${ }^{73}$ A more recent meta-analysis, which assessed the role of bevacizumab in VTE development, included 6,055 patients from ten randomized trials. ${ }^{74}$ No significant increase in the risk of VTE associated with bevacizumab was found, and the lack of VTE risk was consistent with all tumor types. ${ }^{74}$ The reason for the disagreement between those two metaanalyses is probably due to differences in the included trials and analytic methods. ${ }^{74,75}$

Similar to bevacizumab-induced arterial thrombosis, a possible related mechanism for bevacizumab-related VTEs might be the anti-VEGF effect of bevacizumab. VEGF is a protective factor for endothelial cells, regulating multiple biological functions, such as the production of vasoactive mediators and the expression of components of the thrombolytic and coagulation pathways. ${ }^{62}$ The disturbance of vascular homeostasis by blocking VEGF might lead to endothelial dysfunction and subsequent VTEs. Furthermore, bevacizumab may increase the release of proinflammatory cytokines, causing activation of the clotting system. ${ }^{76}$ However, the difference in the bevacizumab-related risk of ATEs and VTEs also implies differences in pathophysiology.

Cancer patients who develop a VTE should be treated according to guidelines recommended by the ESMO. ${ }^{77}$ As in noncancer patients, low molecular weight heparin (LMWH) should be initiated at a dose of $200 \mathrm{U} / \mathrm{kg}$ once daily or $100 \mathrm{U} / \mathrm{kg}$ twice daily for 5-7 days. However, in cancer patients, continuation of LMWH, instead of substitution with vitamin K antagonists, has been proven beneficial. ${ }^{77}$ The majority of patients treated with bevacizumab suffer from metastatic cancer; for those patients, indefinite treatment with LMWH at a dose of $75 \%-80 \%$ of the initial dose is recommended. ${ }^{77}$

\section{Adjunctive effect of radiotherapy}

It has been proposed that the combination of antiangiogenesis agents with radiotherapy might have a synergistic effect through several mechanisms. ${ }^{78}$ Antiangiogenesis inhibitors improve intratumoral oxygenation through normalization of intratumoral vasculature after radiation treatment, leading to increased blood perfusion and distribution of chemotherapy within the tumor; ${ }^{79,80}$ furthermore, improved oxygenation of the tumor induces the generation of reaction oxygen species that damage DNA and interact with the cell membrane to trigger apoptosis. ${ }^{81}$ In addition, treatment with antiangiogenesis agents downregulates growth factors, such as VEGF and EGF that have been shown to mediate radioresistance. ${ }^{82,83} \mathrm{In}$ several clinical trials, the adjunctive effect of radiotherapy to angiogenesis inhibitors has been shown to increase toxicity. In a recent Phase III trial evaluating the use of bevacizumab in combination with radiotherapy and temozolomide for the treatment of newly diagnosed glioblastoma, the incidence rates of both HTN and ATEs were higher in the bevacizumab-radiation arm compared to the control arm (39.3\% vs $12.7 \%$ and $5.9 \%$ vs $1.6 \%$, respectively). ${ }^{84}$ On the other hand, the addition of bevacizumab to radiotherapy in Phase II head and neck and cervical cancer clinical trials has not been shown to exacerbate cardiotoxicity. ${ }^{31-33,85}$

\section{Conclusion}

Through the years, the discovery of novel active antineoplastic agents has dramatically increased the survival of cancer patients, albeit while increasing the incidence of adverse events induced by anticancer treatment. Cardiovascular toxicity in oncology patients is an issue of major importance, particularly because of the diversity of mechanisms involved and the lack of specific treatment guidelines. Bevacizumab, a monoclonal human antibody directed against VEGF, has emerged as a powerful tool in many malignancies, but it has been associated with a variety of cardiac events. On the other hand, several other molecular targeted therapies, such as trastuzumab, as well as traditional chemotherapy drugs, such as anthracyclines, can also cause significant cardiotoxicity. Early recognition of cardiac complications and successful management of these disorders in order to increase the safety of anticancer treatments requires close cooperation of cardiology and oncology specialists.

\section{Disclosure}

The authors report no conflicts of interest in this work.

\section{References}

1. Yeh ET, Tong AT, Lenihan DJ, et al. Cardiovascular complications of cancer therapy: diagnosis, pathogenesis, and management. Circulation. 2004;109(25):3122-3131. 
2. Albini A, Pennesi G, Donatelli F, Cammarota R, De Flora S, Noonan DM. Cardiotoxicity of anticancer drugs: the need for cardio-oncology and cardio-oncological prevention. $J$ Natl Cancer Inst. 2010;102(1): $14-25$.

3. Institute NC. Cancer Therapy Evaluation Program. Available from: http://ctep.cancer.gov/protocoldevelopment/electronic_applications/ docs/ctcv20_4e30e992.pdf. Accessed.

4. Suter TM, Ewer MS. Cancer drugs and the heart: importance and management. Eur Heart J. 2013;34(15):1102-1111.

5. Ewer MS, Lippman SM. Type II chemotherapy-related cardiac dysfunction: time to recognize a new entity. J Clin Oncol. 2005;23(13): 2900-2902.

6. Ferrara N, Hillan KJ, Novotny W. Bevacizumab (Avastin), a humanized anti-VEGF monoclonal antibody for cancer therapy. Biochem Biophys Res Commun. 2005;333(2):328-335.

7. Hurwitz H, Fehrenbacher L, Novotny W, et al. Bevacizumab plus irinotecan, fluorouracil, and leucovorin for metastatic colorectal cancer. N Engl J Med. 2004;350(23):2335-2342.

8. Giantonio BJ, Catalano PJ, Meropol NJ, et al; Eastern Cooperative Oncology Group Study E3200. Bevacizumab in combination with oxaliplatin, fluorouracil, and leucovorin (FOLFOX4) for previously treated metastatic colorectal cancer: results from the Eastern Cooperative Oncology Group Study E3200. J Clin Oncol. 2007;25(12): 1539-1544.

9. Sandler A, Gray R, Perry MC, et al. Paclitaxel-carboplatin alone or with bevacizumab for non-small-cell lung cancer. $N$ Engl J Med. 2006; 355(24):2542-2550.

10. Miller K, Wang M, Gralow J, et al. Paclitaxel plus bevacizumab versus paclitaxel alone for metastatic breast cancer. $N$ Engl J Med. 2007;357(26):2666-2676.

11. Aghajanian C, Blank SV, Goff BA, et al. OCEANS: a randomized, double-blind, placebo-controlled phase III trial of chemotherapy with or without bevacizumab in patients with platinum-sensitive recurrent epithelial ovarian, primary peritoneal, or fallopian tube cancer. $J$ Clin Oncol. 2012;30(17):2039-2045.

12. Friedman HS, Prados MD, Wen PY, et al. Bevacizumab alone and in combination with irinotecan in recurrent glioblastoma. J Clin Oncol. 2009;27(28):4733-4740

13. Rini BI, Halabi S, Rosenberg JE, et al. Phase III trial of bevacizumab plus interferon alfa versus interferon alfa monotherapy in patients with metastatic renal cell carcinoma: final results of CALGB 90206. J Clin Oncol. 2010;28(13):2137-2143.

14. Tewari KS, Sill MW, Long HJ 3rd, et al. Improved survival with bevacizumab in advanced cervical cancer. $N$ Engl J Med. 2014;370(8): 734-743.

15. Galfrascoli E, Piva S, Cinquini M, et al; ORION Collaborative Group. Risk/benefit profile of bevacizumab in metastatic colon cancer: a systematic review and meta-analysis. Dig Liver Dis. 2011;43(4): 286-294.

16. Yang JC, Haworth L, Sherry RM, et al. A randomized trial of bevacizumab, an anti-vascular endothelial growth factor antibody, for metastatic renal cancer. $N$ Engl J Med. 2003;349(5):427-434.

17. Blackwell K, Hurwitz H, Liebérman G, et al. Circulating D-dimer levels are better predictors of overall survival and disease progression than carcinoembryonic antigen levels in patients with metastatic colorectal carcinoma. Cancer. 2004;101(1):77-82.

18. Kamba T, McDonald DM. Mechanisms of adverse effects of anti-VEGF therapy for cancer. Br J Cancer. 2007;96(12):1788-1795.

19. Mir O, Mouthon L, Alexandre J, et al. Bevacizumab-induced cardiovascular events: a consequence of cholesterol emboli syndrome? J Natl Cancer Inst. 2007;99(1):85-86.

20. Gordon MS, Margolin K, Talpaz M, et al. Phase I safety and pharmacokinetic study of recombinant human anti-vascular endothelial growth factor in patients with advanced cancer. J Clin Oncol. 2001;19(3): $843-850$.

21. Siegel AB, Cohen EI, Ocean A, et al. Phase II trial evaluating the clinical and biologic effects of bevacizumab in unresectable hepatocellular carcinoma. J Clin Oncol. 2008;26(18):2992-2998.
22. Cannistra SA, Matulonis UA, Penson RT, et al. Phase II study of bevacizumab in patients with platinum-resistant ovarian cancer or peritoneal serous cancer. J Clin Oncol. 2007;25(33):5180-5186.

23. Burger RA, Sill MW, Monk BJ, Greer BE, Sorosky JI. Phase II trial of bevacizumab in persistent or recurrent epithelial ovarian cancer or primary peritoneal cancer: a Gynecologic Oncology Group Study. J Clin Oncol. 2007;25(33):5165-5171.

24. D'Adamo DR, Anderson SE, Albritton K, et al. Phase II study of doxorubicin and bevacizumab for patients with metastatic soft-tissue sarcomas. J Clin Oncol. 2005;23(28):7135-7142.

25. Emmanouilides C, Sfakiotaki G, Androulakis N, et al. Front-line bevacizumab in combination with oxaliplatin, leucovorin and 5-fluorouracil (FOLFOX) in patients with metastatic colorectal cancer: a multicenter phase II study. BMC Cancer. 2007;7:91.

26. Hainsworth JD, Spigel DR, Sosman JA, et al. Treatment of advanced renal cell carcinoma with the combination bevacizumab/erlotinib/imatinib: a phase I/II trial. Clin Genitourin Cancer. 2007;5(7):427-432.

27. Kindler HL, Friberg G, Singh DA, et al. Phase II trial of bevacizumab plus gemcitabine in patients with advanced pancreatic cancer. $J$ Clin Oncol. 2005;23(31):8033-8040.

28. Meyerhardt JA, Stuart K, Fuchs CS, et al. Phase II study of FOLFOX, bevacizumab and erlotinib as first-line therapy for patients with metastatic colorectal cancer. Ann Oncol. 2007;18(7):1185-1189.

29. Ramaswamy B, Elias AD, Kelbick NT, et al. Phase II trial of bevacizumab in combination with weekly docetaxel in metastatic breast cancer patients. Clin Cancer Res. 2006;12(10):3124-3129.

30. Salama JK, Haraf DJ, Stenson KM, et al. A randomized phase II study of 5-fluorouracil, hydroxyurea, and twice-daily radiotherapy compared with bevacizumab plus 5-fluorouracil, hydroxyurea, and twice-daily radiotherapy for intermediate-stage and T4N0-1 head and neck cancers. Ann Oncol. 2011;22(10):2304-2309.

31. Yoo DS, Kirkpatrick JP, Craciunescu O, et al. Prospective trial of synchronous bevacizumab, erlotinib, and concurrent chemoradiation in locally advanced head and neck cancer. Clin Cancer Res. 2012;18(5): 1404-1414.

32. Fury MG, Lee NY, Sherman E, et al. A phase 2 study of bevacizumab with cisplatin plus intensity-modulated radiation therapy for stage III/IVB head and neck squamous cell cancer. Cancer. 2012; 118(20):5008-5014.

33. Yao M, Galanopoulos N, Lavertu P, et al. Phase II study of bevacizumab in combination with docetaxel and radiation in locally advanced squamous cell carcinoma of the head and neck. Head Neck. Epub June 21, 2014.

34. Argiris A, Kotsakis AP, Hoang T, et al. Cetuximab and bevacizumab: preclinical data and phase II trial in recurrent or metastatic squamous cell carcinoma of the head and neck. Ann Oncol. 2013;24(1):220-225.

35. Cohen EE, Davis DW, Karrison TG, et al. Erlotinib and bevacizumab in patients with recurrent or metastatic squamous-cell carcinoma of the head and neck: a phase I/II study. Lancet Oncol. 2009;10(3):247-257.

36. An MM, Zou Z, Shen H, et al. Incidence and risk of significantly raised blood pressure in cancer patients treated with bevacizumab: an updated meta-analysis. Eur J Clin Pharmacol. 2010;66(8):813-821.

37. Kabbinavar FF, Schulz J, McCleod M, et al. Addition of bevacizumab to bolus fluorouracil and leucovorin in first-line metastatic colorectal cancer: results of a randomized phase II trial. J Clin Oncol. 2005;23(16):3697-3705.

38. Zhu X, Wu S, Dahut WL, Parikh CR. Risks of proteinuria and hypertension with bevacizumab, an antibody against vascular endothelial growth factor: systematic review and meta-analysis. Am J Kidney Dis. 2007;49(2):186-193.

39. Grothey A, Sugrue MM, Purdie DM, et al. Bevacizumab beyond first progression is associated with prolonged overall survival in metastatic colorectal cancer: results from a large observational cohort study (BRiTE). J Clin Oncol. 2008;26(33):5326-5334.

40. Gampenrieder SP, Romeder F, Muß C, et al. Hypertension as a predictive marker for bevacizumab in metastatic breast cancer: results from a retrospective matched-pair analysis. Anticancer Res. 2014;34(1): 227-233. 
41. De Stefano A, Carlomagno C, Pepe S, Bianco R, De Placido S. Bevacizumab-related arterial hypertension as a predictive marker in metastatic colorectal cancer patients. Cancer Chemother Pharmacol. 2011;68(5):1207-1213.

42. Österlund P, Soveri LM, Isoniemi H, Poussa T, Alanko T, Bono P. Hypertension and overall survival in metastatic colorectal cancer patients treated with bevacizumab-containing chemotherapy. $\mathrm{Br} J$ Cancer. 2011;104(4):599-604.

43. Mir O, Coriat R, Cabanes L, et al. An observational study of bevacizumabinduced hypertension as a clinical biomarker of antitumor activity. Oncologist. 2011;16(9):1325-1332.

44. Scartozzi M, Galizia E, Chiorrini S, et al. Arterial hypertension correlates with clinical outcome in colorectal cancer patients treated with first-line bevacizumab. Ann Oncol. 2009;20(2):227-230.

45. Zhong J, Ali AN, Voloschin AD, et al. Bevacizumab-induced hypertension is a predictive marker for improved outcomes in patients with recurrent glioblastoma treated with bevacizumab. Cancer. Epub December 29, 2014.

46. Horsley L, Marti K, Jayson GC. Is the toxicity of anti-angiogenic drugs predictive of outcome? A review of hypertension and proteinuria as biomarkers of response to anti-angiogenic therapy. Expert Opin Drug Metab Toxicol. 2012;8(3):283-293.

47. Mancia G, Fagard R, Narkiewicz K, et al. 2013 ESH/ESC guidelines for the management of arterial hypertension: the Task Force for the Management of Arterial Hypertension of the European Society of Hypertension (ESH) and of the European Society of Cardiology (ESC). Eur Heart J. 2013;34(28):2159-2219.

48. Chobanian AV, Bakris GL, Black HR, et al; Joint National Committee on Prevention, Detection, Evaluation, and Treatment of High Blood Pressure. National Heart, Lung, and Blood Institute; National High Blood Pressure Education Program Coordinating Committee. Seventh report of the Joint National Committee on Prevention, Detection, Evaluation, and Treatment of High Blood Pressure. Hypertension. 2003;42(6):1206-1252.

49. Go AS, Bauman MA, Coleman King SM, et al. An effective approach to high blood pressure control: a science advisory from the American Heart Association, the American College of Cardiology, and the Centers for Disease Control and Prevention. J Am Coll Cardiol. 2014;63(12): 1230-1238.

50. Curigliano G, Cardinale D, Suter T, et al; ESMO Guidelines Working Group. Cardiovascular toxicity induced by chemotherapy, targeted agents and radiotherapy: ESMO Clinical Practice Guidelines. Ann Oncol. 2012;23 Suppl 7:vii155-vii166.

51. Dincer M, Altundag K. Angiotensin-converting enzyme inhibitors for bevacizumab-induced hypertension. Ann Pharmacother. 2006;40(12): 2278-2279.

52. Pande A, Lombardo J, Spangenthal E, Javle M. Hypertension secondary to anti-angiogenic therapy: experience with bevacizumab. Anticancer Res. 2007;27(5B):3465-3470.

53. Izzedine H, Ederhy S, Goldwasser F, et al. Management of hypertension in angiogenesis inhibitor-treated patients. Ann Oncol. 2009;20(5): $807-815$.

54. Chen MH, Kerkelä R, Force T. Mechanisms of cardiac dysfunction associated with tyrosine kinase inhibitor cancer therapeutics. Circulation. 2008;118(1):84-95.

55. Shiojima I, Sato K, Izumiya Y, et al. Disruption of coordinated cardiac hypertrophy and angiogenesis contributes to the transition to heart failure. J Clin Invest. 2005;115(8):2108-2118.

56. Miller KD, Chap LI, Holmes FA, et al. Randomized phase III trial of capecitabine compared with bevacizumab plus capecitabine in patients with previously treated metastatic breast cancer. J Clin Oncol. 2005;23(4):792-799.

57. Hurwitz H, Kabbinavar F. Bevacizumab combined with standard fluoropyrimidine-based chemotherapy regimens to treat colorectal cancer. Oncology. 2005;69 Suppl 3:17-24.

58. Dai F, Shu L, Bian Y, et al. Safety of bevacizumab in treating metastatic colorectal cancer: a systematic review and meta-analysis of all randomized clinical trials. Clin Drug Investig. 2013;33(11):779-788.
59. McMurray JJ, Adamopoulos S, Anker SD, et al; Task Force for the Diagnosis and Treatment of Acute and Chronic Heart Failure 2012 of the European Society of Cardiology; ESC Committee for Practice Guidelines. ESC guidelines for the diagnosis and treatment of acute and chronic heart failure 2012: The Task Force for the Diagnosis and Treatment of Acute and Chronic Heart Failure 2012 of the European Society of Cardiology. Developed in collaboration with the Heart Failure Association (HFA) of the ESC. Eur J Heart Fail. 2012;14(8): 803-869.

60. Hunt SA, Abraham WT, Chin MH, et al; American College of Cardiology Foundation; American Heart Association. 2009 Focused update incorporated into the ACC/AHA 2005 Guidelines for the Diagnosis and Management of Heart Failure in Adults A Report of the American College of Cardiology Foundation/American Heart Association Task Force on Practice Guidelines Developed in Collaboration With the International Society for Heart and Lung Transplantation. J Am Coll Cardiol. 2009;53(15):e1-e90.

61. Kuenen BC, Levi M, Meijers JC, et al. Analysis of coagulation cascade and endothelial cell activation during inhibition of vascular endothelial growth factor/vascular endothelial growth factor receptor pathway in cancer patients. Arterioscler Thromb Vasc Biol. 2002;22(9): $1500-1505$.

62. Kilickap S, Abali H, Celik I. Bevacizumab, bleeding, thrombosis, and warfarin. J Clin Oncol. 2003;21(18):3542; author reply 3543.

63. Scappaticci FA, Skillings JR, Holden SN, et al. Arterial thromboembolic events in patients with metastatic carcinoma treated with chemotherapy and bevacizumab. J Natl Cancer Inst. 2007;99(16):1232-1239.

64. Ranpura V, Hapani S, Chuang J, Wu S. Risk of cardiac ischemia and arterial thromboembolic events with the angiogenesis inhibitor bevacizumab in cancer patients: a meta-analysis of randomized controlled trials. Acta Oncol. 2010;49(3):287-297.

65. Tsai HT, Marshall JL, Weiss SR, et al. Bevacizumab use and risk of cardiovascular adverse events among elderly patients with colorectal cancer receiving chemotherapy: a population-based study. Ann Oncol. 2013;24(6):1574-1579.

66. Steg PG, James SK, Atar D, et al; Task Force on the management of ST-segment elevation acute myocardial infarction of the European Society of Cardiology (ESC). ESC Guidelines for the management of acute myocardial infarction in patients presenting with ST-segment elevation. Eur Heart J. 2012;33(20):2569-2619.

67. Hamm CW, Bassand JP, Agewall S, et al; ESC Committee for Practice Guidelines. ESC Guidelines for the management of acute coronary syndromes in patients presenting without persistent ST-segment elevation: The Task Force for the management of acute coronary syndromes (ACS) in patients presenting without persistent ST-segment elevation of the European Society of Cardiology (ESC). Eur Heart J. 2011;32(23):2999-3054.

68. Sarkiss MG, Yusuf SW, Warneke CL, et al. Impact of aspirin therapy in cancer patients with thrombocytopenia and acute coronary syndromes. Cancer. 2007;109(3):621-627.

69. Kabbinavar F, Hurwitz HI, Fehrenbacher L, et al. Phase II, randomized trial comparing bevacizumab plus fluorouracil (FU)/leucovorin (LV) with FU/LV alone in patients with metastatic colorectal cancer. $J$ Clin Oncol. 2003;21(1):60-65.

70. Escudier B, Pluzanska A, Koralewski P, et al; AVOREN Trial investigators. Bevacizumab plus interferon alfa-2a for treatment of metastatic renal cell carcinoma: a randomised, double-blind phase III trial. Lancet. 2007;370(9605):2103-2111.

71. Johnson DH, Fehrenbacher L, Novotny WF, et al. Randomized phase II trial comparing bevacizumab plus carboplatin and paclitaxel with carboplatin and paclitaxel alone in previously untreated locally advanced or metastatic non-small-cell lung cancer. J Clin Oncol. 2004;22(11):2184-2191.

72. Nalluri SR, Chu D, Keresztes R, Zhu X, Wu S. Risk of venous thromboembolism with the angiogenesis inhibitor bevacizumab in cancer patients: a meta-analysis. JAMA. 2008;300(19):2277-2285.

73. Minor DR. Risk of venous thromboembolism with bevacizumab in cancer patients. JAMA. 2009;301(14):1434; author reply 1435-1436. 
74. Hurwitz HI, Saltz LB, Van Cutsem E, et al. Venous thromboembolic events with chemotherapy plus bevacizumab: a pooled analysis of patients in randomized phase II and III studies. J Clin Oncol. 2011; 29(13):1757-1764.

75. Hapani S, Wu S. Controversial role of bevacizumab in the development of venous thromboembolic events. J Clin Oncol. 2011;29(25):3490; author reply 3490-3491.

76. Hesser BA, Liang XH, Camenisch G, et al. Down syndrome critical region protein 1 (DSCR1), a novel VEGF target gene that regulates expression of inflammatory markers on activated endothelial cells. Blood. 2004;104(1):149-158.

77. Mandalà M, Falanga A, Roila F; ESMO Guidelines Working Group. Management of venous thromboembolism (VTE) in cancer patients: ESMO Clinical Practice Guidelines. Ann Oncol. 2011;22 Suppl 6: vi85-vi92.

78. Vassilakopoulou M, Psyrri A, Argiris A. Targeting angiogenesis in head and neck cancer. Oral Oncol. 2015;51(5):409-415.

79. Jain RK. Normalizing tumor vasculature with anti-angiogenic therapy: a new paradigm for combination therapy. Nat Med. 2001;7(9): 987-989.
80. Teicher BA, Holden SA, Ara G, et al. Potentiation of cytotoxic cancer therapies by TNP-470 alone and with other anti-angiogenic agents. Int J Cancer. 1994;57(6):920-925.

81. Koch S, Claesson-Welsh L. Signal transduction by vascular endothelial growth factor receptors. Cold Spring Harb Perspect Med. 2012;2(7): a006502.

82. Sheridan MT, O’Dwyer T, Seymour CB, Mothersill CE. Potential indicators of radiosensitivity in squamous cell carcinoma of the head and neck. Radiat Oncol Investig. 1997;5(4):180-186.

83. Tanno S, Yanagawa N, Habiro A, et al. Serine/threonine kinase AKT is frequently activated in human bile duct cancer and is associated with increased radioresistance. Cancer Res. 2004;64(10):3486-3490.

84. Chinot OL, Wick W, Mason W, et al. Bevacizumab plus radiotherapytemozolomide for newly diagnosed glioblastoma. $N \mathrm{Engl} \mathrm{J} \mathrm{Med.}$ 2014;370(8):709-722.

85. Schefter T, Winter K, Kwon JS, et al; Radiation Therapy Oncology Group (RTOG). RTOG 0417: efficacy of bevacizumab in combination with definitive radiation therapy and cisplatin chemotherapy in untreated patients with locally advanced cervical carcinoma. Int J Radiat Oncol Biol Phys. 2014;88(1):101-105.
Cancer Management and Research

\section{Publish your work in this journal}

Cancer Management and Research is an international, peer-reviewed open access journal focusing on cancer research and the optimal use of preventative and integrated treatment interventions to achieve improved outcomes, enhanced survival and quality of life for the cancer patient The journal welcomes original research, clinical \& epidemiological

\section{Dovepress}

studies, reviews \& evaluations, guidelines, expert opinion \& commentary, case reports \& extended reports. The manuscript management system is completely online and includes a very quick and fair peerreview system, which is all easy to use. Visit http://www.dovepress.com/ testimonials.php to read real quotes from published authors. 